\title{
Adaptive Backstepping Sliding-Mode Control of the Electronic Throttle System in Modern Automobiles
}

\author{
Rui Bai and Shaocheng Tong \\ School of Electrical Engineering, Liaoning University of Technology, Jinzhou 121001, China \\ Correspondence should be addressed to Rui Bai; broffice@126.com
}

Received 19 December 2013; Revised 25 March 2014; Accepted 28 March 2014; Published 24 April 2014

Academic Editor: Mingcong Deng

Copyright ( 2014 R. Bai and S. Tong. This is an open access article distributed under the Creative Commons Attribution License, which permits unrestricted use, distribution, and reproduction in any medium, provided the original work is properly cited.

\begin{abstract}
In modern automobiles, electronic throttle is a DC-motor-driven valve that regulates air inflow into the vehicle's combustion system. The electronic throttle is increasingly being used in order to improve the vehicle drivability, fuel economy, and emissions. Electronic throttle system has the nonlinear dynamical characteristics with the unknown disturbance and parameters. At first, the dynamical nonlinear model of the electronic throttle is built in this paper. Based on the model and using the backstepping design technique, a new adaptive backstepping sliding-mode controller of the electronic throttle is developed. During the backstepping design process, parameter adaptive law is designed to estimate the unknown parameter, and sliding-mode control term is applied to compensate the unknown disturbance. The proposed controller can make the actual angle of the electronic throttle track its set point with the satisfactory performance. Finally, a computer simulation is performed, and simulation results verify that the proposed control method can achieve favorable tracking performance.
\end{abstract}

\section{Introduction}

In recent years, many important functions of modern automobiles are shifting from a purely mechanical to an electromechanical implementation. These functions are implemented by using the so-called "x-by-wire" systems, including drive-by-wire and steer-by-wire systems $[1,2]$. Electronic throttle system is one of the important drive-by-wire systems for modern automobiles. The electronic throttle is essentially a DC-motor-driven valve that regulates air inflow into the vehicle's combustion system. Its control system positions the throttle valve according to the reference opening angle which is provided by the engine control unit. In recent years, the electronic throttle is increasingly being used in modern automobiles in order to improve the vehicle drivability, fuel economy, and emissions.

In fact, electronic throttle control is a typical tracking problem of nonlinear control systems. Nonlinear control scheme has been attracting more and more attention from the researchers almost in every field of the control design systems $[3,4]$. There are many nonlinear control schemes that have been proposed and studied. For example, in [57], based on robust right coprime factorization, tracking problem of nonlinear feedback control systems is considered and studied. The proposed universal design scheme on the nonlinear control system can not only guarantee the robust stability of the perturbed systems but also realize the output tracking to the reference input. Sliding-mode control [8] and backstepping control [9] are also some widely utilized nonlinear control schemes. All these nonlinear control methods mentioned above have been widely used in practical applications due to their robustness.

The synthesis of a satisfactory electronic throttle control (ETC) system is difficult due to the presence of the strong nonlinear characteristic, unknown parameter, and disturbance. This challenging control problem has attracted significant attention of the research community and automotive industry in recent years. In [2], a new intelligent fuzzy controller is proposed. It can handle the nonlinear hysteretic of electronic throttle. In [10], the controller synthesis is performed in discrete time by solving a constrained timeoptimal control problem of the throttle. In [11], the dynamic programming (DP) technique is also used to obtain the optimal gear shift and throttle opening angle which maximizes fuel economy while satisfying the power demand. In [12], 
an integrated control strategy is proposed, which consists of a proportional-integral-derivative (PID) controller and a feedback compensator for friction and limp-home effects. In $[13,14]$, some novel approximate model-based robust nonlinear control strategies are presented for the electronic throttle valve. In [15], a throttle control algorithm for improving the engine response is proposed, and the proposed control algorithm can compensate two delays that existed in the throttle response and manifold filling.

Sliding-mode control (SMC) has been widely used in practical applications due to its robustness against parameter variations and disturbances $[8,16]$. There are also some works that introduce sliding-mode control to control the electronic throttle. In [17], a cascade control structure of the electronic throttle is presented and discussed, which is composed of an inner current sliding-mode controller, an intermediate velocity sliding-mode controller, and an outer position digital linear controller. In [18], the nonlinear hysteretic characteristic of the electronic throttle is described and the sliding-mode control method is proposed to control the electronic throttle. References $[19,20]$ designed an observer-based sliding-mode controller based on an identified model for a drive-bywire throttle control system. In [21], a neural network-based sliding-mode controller for an electronic throttle is proposed, in which a static neural network is used as an estimator of the state-dependent uncertainties in the system. In [22], the control of an electronic throttle valve based on secondorder sliding-mode concepts is presented, and the twisting algorithm is chosen as the control law.

Although the above mentioned control methods can achieve the acceptable control performance, there are also some shortcomings in these existing control methods. These control methods have complex structure and design process. As we know, the complex design process of the controller often leads to the difficulty of its realization in the actual automotive manufacturing industry. Meanwhile, the characteristics including the unknown parameter and disturbance of the electronic throttle are not fully considered and processed in these existing control methods.

Backstepping technique is a systematic design scheme. Its main idea is to decompose a complex system into multiple small-scale subsystems, then to design recursively control Lyapunov function and virtual controller for each subsystem, and finally obtain the original control law [9, 23, 24]. For the systematic design process, backstepping control scheme is easy to be realized and it has been applied in many practical applications cases, such as induction motor [25], chemical process [26], and robot manipulator [27].

Motivated by the advantage of the backstepping design method, this paper investigates the backstepping control problem of the electronic throttle. A new adaptive backstepping sliding-mode controller of the electronic throttle is developed in this paper. During the backstepping design process, parameter adaptive law is designed to estimate the unknown parameter, and sliding-mode term is applied to compensate the unknown disturbance. The proposed adaptive backstepping sliding-mode controller can achieve the satisfactory performance; that is, the actual angle of the electronic throttle can track its set point. Finally, a computer simulation is performed, and simulation results verify the effectiveness of the proposed control method.

It should be noted that the adaptive backstepping slidingmode control method has not been utilized for the electronic throttle system in the existing references. This paper proposes and designs an adaptive backstepping sliding-mode controller for the electronic throttle. The proposed control method is suitable for the characteristics of the electronic throttle system, and the satisfactory control performance also can be obtained. Therefore, the new control strategy of the electronic throttle in this paper is valuable for the modern automobile industry.

This paper is organized as follows: Section 2 describes the nonlinear mathematical model of the electronic throttle. Section 3 designs the adaptive backstepping sliding-mode controller of the electronic throttle. Section 4 illustrates the simulation results and finally Section 5 shows the conclusion of this paper.

\section{Descriptions and Nonlinear Model of the Electronic Throttle}

There are some symbols in this section. At first, definitions of these symbols are described as follows:

$\theta_{r}$ : set point of the valve plate angle,

$\theta(t)$ : actual angle of the valve plate,

$\theta_{0}$ : static angle of the valve plate,

$\omega(t)$ : angle speed of the valve plate,

$i_{a}(t)$ : armature current,

$R_{a}$ : armature resistance,

$U_{a}(t)$ : input voltage of the motor,

$U_{b}(t)$ : electromotive force,

$U_{b a t}$ : supply voltage,

$D(t)$ : duty cycle of the bipolar chopper,

$T_{e}(t)$ : electromagnetism torque,

$T_{L}(t)$ : load torque of the DC motor,

$T_{s}(t)$ : return spring torque,

$T_{f}(t)$ : friction torque,

$K_{t}$ : torque constant,

$K_{b}$ : electromotive force constant,

$K_{s}$ : elastic coefficient,

$K_{m}$ : torque compensation coefficient,

$K_{d}$ : sliding friction coefficient,

$K_{k}$ : coulomb friction coefficient,

$J:$ moment of inertia,

$G_{r}$ : gear ratio. 


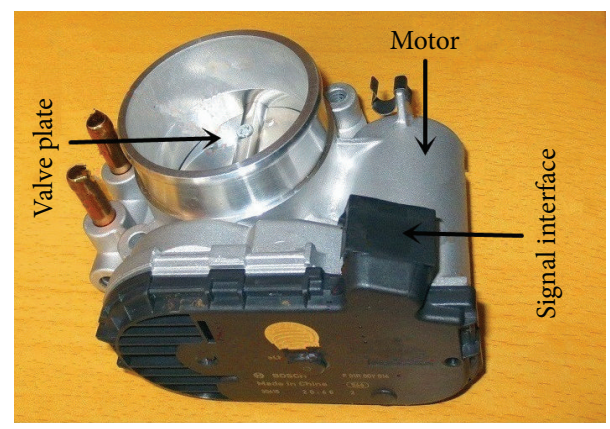

Figure 1: External view.

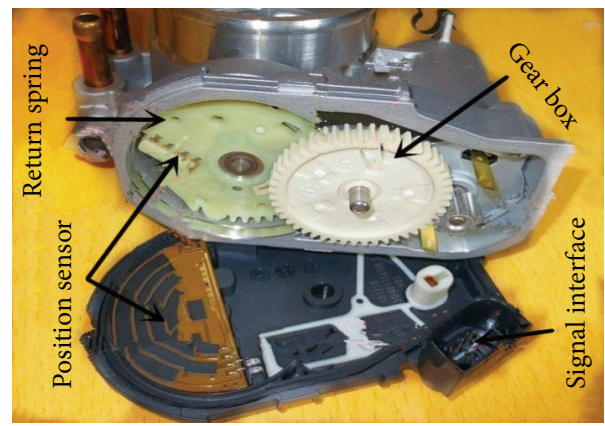

FIGURE 2: Internal view.

2.1. Description of the Electronic Throttle. The real figures of the electronic throttle are shown in Figures 1 and 2. Figure 1 is the external view of the electronic throttle. Figure 2 is the internal view of the electronic throttle. The schematic of a typical electronic throttle control system is shown in Figure 3.

There are a controller, a bipolar chopper, and an electronic throttle body (ETB) in Figure 3. ETB consists of a DC drive powered by the bipolar chopper, a gearbox, a valve plate, a return spring, and a position sensor. When the valve plate angle is regulated, the air inflow into the vehicle's combustion system can also be regulated. The control objective of the electronic throttle is to control the valve plate angle tracking its set point with the satisfactory performance.

2.2. Nonlinear Model of the Electronic Throttle. In this section, we establish the nonlinear model of the electronic throttle. At first, we build the motion equation of the electronic throttle system. According to the motor motion theory, the motion equation of the electronic throttle is

$$
G_{r} T_{e}(t)-T_{L}(t)=G_{r}^{2} J \frac{d \omega(t)}{d t} .
$$

In the electronic throttle, the driving motor is $\mathrm{DC}$ motor. The electromagnetism torque $T_{e}(t)$ of DC motor is determined mainly by the armature current. Computation formula of $T_{e}(t)$ is

$$
T_{e}(t)=K_{t} i_{a}(t)
$$

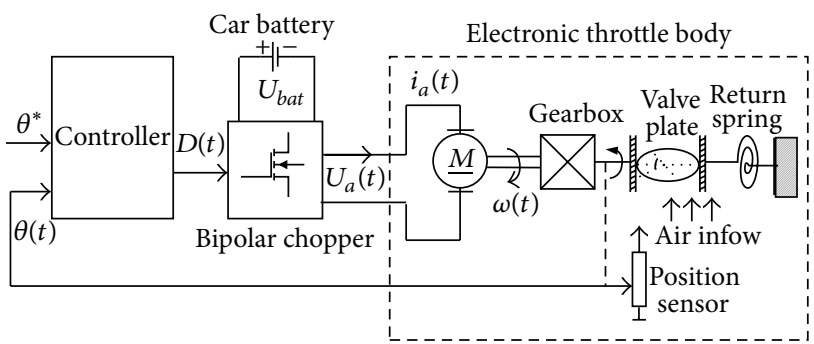

FIgURE 3: Electronic throttle control system.

In the motor armature circuit, the relation between current $i_{a}(t)$ and input voltage $U_{a}(t)$ is described as

$$
i_{a}(t) R_{a}=U_{a}(t)-U_{b}(t),
$$

where

$$
\begin{gathered}
U_{a}(t)=U_{b a t} \times D(t), \\
U_{b}(t)=K_{b} \times G_{r} \times \omega(t) .
\end{gathered}
$$

By substituting (4) into (3), we have

$$
i_{a}(t)=\frac{U_{b a t} \times D(t)-K_{b} \times G_{r} \times \omega(t)}{R_{a}} .
$$

By substituting (5) into (2), we get

$$
T_{e}(t)=K_{t} \frac{U_{b a t} \times D(t)-K_{b} \times G_{r} \times \omega(t)}{R_{a}} .
$$

Therefore, (6) is the expression of $T_{e}(t)$.

In the following steps, we will deduce the detailed expression of $T_{L}(t)$. From the structure of the electronic throttle, we know that the load torque $T_{L}(t)$ in the electronic throttle mainly consists of the return spring torque $T_{s}(t)$ and the friction torque $T_{f}(t)$ :

$$
T_{L}(t)=T_{s}(t)+T_{f}(t) .
$$

According to the mechanism analysis, the nonlinear return spring torque $T_{s}(t)$ is

$$
T_{s}(t)=K_{s}\left(\theta(t)-\theta_{0}\right)+K_{m} \operatorname{sgn}\left(\theta(t)-\theta_{0}\right) .
$$

Friction torque $T_{f}(t)$ is composed of the static and coulomb frictions, which is shown as follows:

$$
T_{f}(t)=K_{d} \omega(t)+K_{k} \operatorname{sgn}(\omega(t)) .
$$

In (9), $K_{d} \omega(t)$ is the static friction and $K_{k} \operatorname{sgn}(\omega(t))$ is the coulomb friction.

By substituting (6)-(9) into (1), we get

$$
\begin{aligned}
\frac{d \omega(t)}{d t}= & -\frac{K_{s}}{G_{r}^{2} \times J} \times\left(\theta(t)-\theta_{0}\right) \\
& -\left(\frac{K_{b} \times K_{t}}{J R_{a}}+\frac{K_{d}}{G_{r}^{2} \times J}\right) \omega(t)+\frac{K_{t} \times U_{b a t} \times D(t)}{G_{r} \times J \times R_{a}} \\
& +\frac{-K_{m} \operatorname{sgn}\left(\theta(t)-\theta_{0}\right)-K_{k} \operatorname{sgn}(\omega(t))}{G_{r}^{2} \times J} .
\end{aligned}
$$


In fact, there are some existing models of the electronic throttle in some references. However, the dynamical characteristics of the electronic throttle cannot be fully reflected by these models due to the simplified modeling process. For example, in $[15,19,28]$, the return spring torque or the friction torque is simplified as linear models. In $[17,18]$, the static friction or coulomb friction is neglected. In this paper, as shown in (7)-(8), the dynamical characteristics of the return spring torque and the friction torque are fully considered at the same time in the modeling process. Therefore, the model in (10) has better performance compared with some existing models.

Defining state variables $x_{1}(t)=\theta(t)-\theta_{0}$ and $x_{2}(t)=\omega(t)$, input variable $u(t)=D(t)$, and the output variable $y(t)=$ $\theta(t)$, we can obtain the dynamical state-space model of the electronic throttle system:

$$
\begin{aligned}
& \dot{x}_{1}(t)= x_{2}(t), \\
& \dot{x}_{2}(t)= \frac{K_{t} \times U_{b a t}}{G_{r} \times J \times R_{a}} u(t)-\frac{K_{s}}{G_{r}^{2} \times J} \\
& \times x_{1}(t)-\left(\frac{K_{b} \times K_{t}}{J R_{a}}+\frac{K_{d}}{G_{r}^{2} \times J}\right) x_{2}(t) \\
&+\frac{-K_{m} \operatorname{sgn}\left(x_{1}(t)\right)-K_{k} \operatorname{sgn}\left(x_{2}(t)\right)}{G_{r}^{2} \times J}, \\
& y(t)=x_{1}(t)+\theta_{0} .
\end{aligned}
$$

In (11), $J, R_{a}, K_{t}, K_{m}$, and $K_{k}$ are the known parameters. $K_{s}$, $K_{b}$, and $K_{d}$ are the unknown parameters.

The main external disturbance signal in the electronic throttle system is the external current disturbance. As shown in Figure 3, the control signal is transformed to a pulse width modulation (PWM) signal by the bipolar chopper that is connected to the DC motor. Frequency fluctuation of PWM often causes the external current disturbance. The accurate value of the external current disturbance is difficult to be measured. However, the maximum range of this external disturbance signal can be determined by some experiments and experiences. In fact, by using some experiments and experiences, we know that the maximum range of external current disturbance is one ampere $(\mathrm{A})$.

Then, letting $\mu_{0}=\left(K_{t} \times U_{b a t}\right) /\left(G_{r} \times J \times R_{a}\right), \mu_{1}=K_{s} /\left(G_{r}^{2} \times\right.$ $J), \mu_{2}=\left(\left(\left(K_{b} \times K_{t}\right) / J R_{a}\right)+\left(K_{d} /\left(G_{r}^{2} \times J\right)\right)\right), F\left(x_{1}, x_{2}, t\right)=$ $\left(-K_{m} \operatorname{sgn}\left(x_{1}(t)\right)-K_{k} \operatorname{sgn}\left(x_{2}(t)\right)\right) /\left(G_{r}^{2} \times J\right)$ and considering the external disturbance, (11) is rewritten as

$$
\begin{aligned}
\dot{x}_{1}(t)= & x_{2}(t), \\
\dot{x}_{2}(t)= & \mu_{0} u(t)-\mu_{1} x_{1}(t)-\mu_{2} x_{2}(t) \\
& +F\left(x_{1}, x_{2}, t\right)+d(t),
\end{aligned}
$$

where $d(t)$ is the external disturbance and satisfies the following condition:

$$
|d(t)| \leq D
$$

where $D$ is a known constant, which represents the maximum value of the external disturbance. In this paper, $D$ can be selected as 1 .

From the definitions of $\mu_{0}, \mu_{1}$, and $\mu_{2}$, we know that $\mu_{0}$ is the known parameter; however, $\mu_{1}$ and $\mu_{2}$ are the unknown parameters in (13).

\section{Adaptive Backstepping Sliding-Mode Control Design}

From (11), we know the output $y(t)$ is the actual angle $\theta(t)$. The control objective of this paper is to design a control system such that the output $y(t)$ of the system shown in (11) can track the set point of the valve plate angle $\theta^{*}$ asymptotically.

Based on the dynamical state-space model and using the backstepping design technique, a new adaptive backstepping sliding-mode controller is developed for the electronic throttle. During the backstepping design process, parameter adaptive law is designed to estimate the unknown parameter and sliding-mode control term is applied to compensate the unknown disturbance. The proposed backstepping control procedure is described step by step as follows.

Step 1. For the position-tracking objective, the tracking error is defined as

$$
z_{1}(t)=x_{1}(t)-x_{r}
$$

where $x_{r}=\theta_{r}-\theta_{0}$.

Take $\alpha(t)$ as a virtual control and define

$$
z_{2}(t)=x_{2}(t)-\alpha(t)
$$

Consider the following Lyapunov function candidate:

$$
V_{1}(t)=\frac{1}{2} z_{1}^{2}(t)
$$

The time derivative of $V_{1}(t)$ is

$$
\dot{V}_{1}(t)=z_{1}(t) \dot{z}_{1}(t) \text {. }
$$

From (15) and (16), we know

$$
\begin{aligned}
\dot{z}_{1}(t) & =\dot{x}_{1}(t) \\
& =x_{2}(t) \\
& =-c_{1} z_{1}(t)+z_{2}(t)+c_{1} z_{1}(t)+\alpha(t) .
\end{aligned}
$$

Choose the virtual control function $\alpha(t)$ as

$$
\alpha(t)=-c_{1} z_{1}(t) .
$$

By substituting (20) into (19), we have

$$
\dot{z}_{1}(t)=-c_{1} z_{1}(t)+z_{2}(t) .
$$

By using (21) and (18), we get

$$
\begin{aligned}
\dot{V}_{1}(t) & =z_{1}(t) \dot{z}_{1}(t) \\
& =z_{1}(t)\left(-c_{1} z_{1}(t)+z_{2}(t)\right) \\
& =-c_{1} z_{1}^{2}(t)+z_{1}(t) z_{2}(t) .
\end{aligned}
$$


From (22), we know if $z_{2}(t)$ is equal to zero, the time derivative of $V_{1}(t)$ will be smaller than or equal to zero. If $\dot{V}_{1}(t) \leq 0$, we know $z_{1}(t)$ will converge to zero and $x_{1}(t)$ will converge to the set point $x_{d}$. Therefore, in the next step, we will design a controller $u(t)$ to make $z_{2}(t)$ converge to zero.

Step 2. In (13), $\mu_{1}$ and $\mu_{2}$ are unknown parameters. At first, we define

$$
\begin{aligned}
& \widetilde{\mu}_{1}=\widehat{\mu}_{1}-\mu_{1}, \\
& \widetilde{\mu}_{2}=\widehat{\mu}_{2}-\mu_{2},
\end{aligned}
$$

where $\widehat{\mu}_{1}$ is the estimation value of $\mu_{1}$ and $\widehat{\mu}_{2}$ is the estimation value of $\mu_{2} \cdot \widetilde{\mu}_{1}$ and $\widetilde{\mu}_{2}$ are the estimation errors. $V_{2}(t)$ :

Consider the following Lyapunov function candidate

$$
V_{2}(t)=\frac{1}{2} z_{2}^{2}(t)+V_{1}(t)+\frac{1}{2 \gamma_{1}} \tilde{\mu}_{1}^{2}+\frac{1}{2 \gamma_{2}} \tilde{\mu}_{2}^{2} .
$$

The time derivative of $V_{2}(t)$ is

$$
\begin{aligned}
\dot{V}_{2}(t)= & z_{2}(t) \dot{z}_{2}(t)+\dot{V}_{1}(t) \\
& +\frac{1}{\gamma_{1}} \dot{\tilde{\mu}}_{1} \tilde{\mu}_{1}+\frac{1}{\gamma_{2}} \dot{\tilde{\mu}}_{2} \widetilde{\mu}_{2} \\
= & z_{2}(t) \dot{z}_{2}(t)-c_{1} z_{1}^{2}(t)+z_{1}(t) z_{2}(t) \\
& +\frac{1}{\gamma_{1}} \dot{\tilde{\mu}}_{1} \tilde{\mu}_{1}+\frac{1}{\gamma_{2}} \dot{\tilde{\mu}}_{2} \widetilde{\mu}_{2} \\
= & z_{2}(t) \dot{z}_{2}(t)-c_{1} z_{1}^{2}(t)+z_{1}(t) z_{2}(t) \\
& +\frac{1}{\gamma_{1}} \dot{\vec{\mu}}_{1}\left(\widehat{\mu}_{1}-\mu_{1}\right)+\frac{1}{\gamma_{2}} \dot{\vec{\mu}}_{2}\left(\widehat{\mu}_{2}-\mu_{2}\right) .
\end{aligned}
$$

According to (13), (16), and (20), we know

$$
\begin{aligned}
\dot{z}_{2}(t)= & \dot{x}_{2}(t)-\dot{\alpha}(t) \\
= & \mu_{0} u(t)-\mu_{1} x_{1}(t)-\mu_{2} x_{2}(t) \\
& +F\left(x_{1}, x_{2}, t\right)+d(t)-\dot{\alpha}(t) \\
= & \mu_{0} u(t)-\mu_{1} x_{1}(t)-\mu_{2} x_{2}(t) \\
& +F\left(x_{1}, x_{2}, t\right)+d(t)+c_{1} x_{2}(t) .
\end{aligned}
$$

By substituting (26) into (25), we have

$$
\begin{aligned}
\dot{V}_{2}(t)= & z_{2}(t) \dot{z}_{2}(t)-c_{1} z_{1}^{2}(t)+z_{1}(t) z_{2}(t) \\
& +\frac{1}{\gamma_{1}} \dot{\hat{\mu}}_{1}\left(\widehat{\mu}_{1}-\mu_{1}\right)+\frac{1}{\gamma_{2}} \dot{\hat{\mu}}_{2}\left(\widehat{\mu}_{2}-\mu_{2}\right) \\
= & z_{2}(t)\left[\mu_{0} u(t)-\mu_{1} x_{1}(t)-\mu_{2} x_{2}(t)\right. \\
& \left.+F\left(x_{1}, x_{2}, t\right)+d(t)+c_{1} x_{2}(t)\right]
\end{aligned}
$$

$$
\begin{aligned}
& \quad-c_{1} z_{1}^{2}(t)+z_{1}(t) z_{2}(t) \\
& +\frac{1}{\gamma_{1}} \dot{\hat{\mu}}_{1}\left(\widehat{\mu}_{1}-\mu_{1}\right)+\frac{1}{\gamma_{2}} \dot{\hat{\mu}}_{2}\left(\widehat{\mu}_{2}-\mu_{2}\right) \\
& =z_{2}(t) \mu_{0} u(t)+z_{2}(t) F\left(x_{1}, x_{2}, t\right) \\
& +z_{2}(t) d(t)+z_{2}(t) c_{1} x_{2}(t)-c_{1} z_{1}^{2}(t) \\
& +z_{1}(t) z_{2}(t)+\frac{1}{\gamma_{1}} \dot{\hat{\mu}}_{1} \widehat{\mu}_{1}+\frac{1}{\gamma_{2}} \dot{\hat{\mu}}_{2} \widehat{\mu}_{2} \\
& +\mu_{1}\left(-z_{2}(t) x_{1}(t)-\frac{1}{\gamma_{1}} \dot{\hat{\mu}}_{1}\right) \\
& +\mu_{2}\left(-z_{2}(t) x_{2}(t)-\frac{1}{\gamma_{2}} \dot{\hat{\mu}}_{2}\right) .
\end{aligned}
$$

We design the parameter adaptive laws as

$$
\begin{aligned}
& \dot{\hat{\mu}}_{1}=-\gamma_{1} z_{2}(t) x_{1}(t), \\
& \dot{\hat{\mu}}_{2}=-\gamma_{2} z_{2}(t) x_{2}(t) .
\end{aligned}
$$

By substituting (28)-(29) into (27), we have

$$
\begin{aligned}
\dot{V}_{2}(t)= & z_{2}(t) \mu_{0} u(t)+z_{2}(t) F\left(x_{1}, x_{2}, t\right) \\
& +z_{2}(t) d(t)+z_{2}(t) c_{1} x_{2}(t)-c_{1} z_{1}^{2}(t) \\
& +z_{1}(t) z_{2}(t)+\frac{1}{\gamma_{1}} \dot{\hat{\mu}}_{1} \widehat{\mu}_{1}+\frac{1}{\gamma_{2}} \dot{\hat{\mu}}_{2} \widehat{\mu}_{2} \\
= & z_{2}(t) \mu_{0} u(t)+z_{2}(t) F\left(x_{1}, x_{2}, t\right) \\
& +z_{2}(t) d(t)+z_{2}(t) c_{1} x_{2}(t) \\
& -c_{1} z_{1}^{2}(t)+z_{1}(t) z_{2}(t) \\
& -z_{2}(t) x_{1}(t) \widehat{\mu}_{1}-z_{2}(t) x_{2}(t) \widehat{\mu}_{2} .
\end{aligned}
$$

Choose the control function $u(t)$ as

$$
\begin{aligned}
u(t)=\frac{1}{\mu_{0}}\{-F & \left(x_{1}, x_{2}, t\right)-c_{1} x_{2}(t) \\
& -\eta \operatorname{sgn}\left(z_{2}(t)\right)+x_{1}(t) \widehat{\mu}_{1} \\
& \left.+x_{2}(t) \widehat{\mu}_{2}-z_{1}(t)-c_{2} z_{2}(t)\right\},
\end{aligned}
$$

where $\eta$ is a constant and satisfies the following condition:

$$
\eta \geq D
$$

In (31), $\left(1 / \mu_{0}\right) \eta \operatorname{sgn}\left(z_{2}(t)\right)$ is the slide-mode control term, which is utilized to compensate the external disturbance $d(t)$. 
By substituting (31) into (30), we have

$$
\begin{aligned}
\dot{V}_{2}(t)= & z_{2}(t)\left\{-F\left(x_{1}, x_{2}, t\right)-c_{1} x_{2}(t)\right. \\
& -\eta \operatorname{sgn}\left(z_{2}(t)\right)+x_{1}(t) \widehat{\mu}_{1} \\
& \left.+x_{2}(t) \widehat{\mu}_{2}-z_{1}(t)-c_{2} z_{2}(t)\right\} \\
& +z_{2}(t) F\left(x_{1}, x_{2}, t\right)+z_{2}(t) d(t) \\
& +z_{2}(t) c_{1} x_{2}(t)-c_{1} z_{1}^{2}(t)+z_{1}(t) z_{2}(t) \\
& -z_{2}(t) x_{1}(t) \widehat{\mu}_{1}-z_{2}(t) x_{2}(t) \widehat{\mu}_{2} \\
= & -c_{1} z_{1}^{2}(t)-c_{2} z_{2}^{2}(t) \\
& -\eta z_{2}(t) \operatorname{sgn}\left(z_{2}(t)\right)+z_{2}(t) d(t) \\
= & -c_{1} z_{1}^{2}(t)-c_{2} z_{2}^{2}(t) \\
& -\eta\left|z_{2}(t)\right|+z_{2}(t) d(t) .
\end{aligned}
$$

According to (32), we know

$$
\dot{V}_{2}(t) \leq-c_{1} z_{1}^{2}(t)-c_{2} z_{2}^{2}(t) \leq 0 .
$$

From (34), we know if we choose the parameter adaptive laws shown in (28)-(29) and controller shown in (31), the variables $z_{1}(t)$ and $z_{2}(t)$ will converge to zero. That is, the output $y(t)=$ $x_{1}(t)$ of the system shown in (12) can track its set point $x_{d}$ asymptotically.

\section{Computer Simulation}

In this section, we perform computer simulation to confirm the effectiveness of the proposed adaptive backstepping sliding-mode controller. It should be noted that some parameters are unknown in the actual electronic throttle system. The unknown parameters are described in Section 2. In the simulation, it is necessary to know the parameters in the dynamical model of the electronic throttle. Therefore, we must give some assumption values for these unknown parameters in the simulation. The values of the parameters in the electronic throttle system in the simulation are given in Table 1.

In the simulation, the external disturbance also should be given. From Section 2, we know that the maximum range of external current disturbance is one ampere (A). Therefore, during the simulation process, we can select the external disturbance as $d(t)=\sin (t)$.

The control algorithm in this simulation is shown as (31). According to (32) and $d(t), \eta$ in (32) can be selected as $\eta=1$.

Simulation results are shown in Figures 4-9. Figure 4 shows the set point of the electronic throttle angle, that is, $x_{d}$. Figure 5 shows the actual angle of the electronic throttle, that is, $x_{1}(t)$. Figure 6 shows the actual angle speed of the electronic throttle, that is, $x_{2}(t)$. Figure 7 shows the estimation value of the first unknown parameter, that is, $\widehat{\mu}_{1}$. Figure 8 shows the estimation value of the second unknown parameter, that is, $\widehat{\mu}_{2}$. Figure 9 shows the duty cycle of the bipolar chopper, that is, the input variable $u(t)=D(t)$ in (12).
TABLE 1: Parameter values.

$$
\begin{aligned}
& G_{r}=17 \\
& K_{b}=0.016 \mathrm{~N} \cdot \mathrm{m} / \mathrm{A} \\
& K_{s}=0.0247 \mathrm{~N} \cdot \mathrm{m} / \mathrm{rad} \\
& \theta_{0}=0.16 \mathrm{rad} \\
& J=4 \times 10^{-6} \mathrm{Kg} \cdot \mathrm{m}^{2} \\
& K_{t}=0.016 \mathrm{~N} \cdot \mathrm{m} / \mathrm{A} \\
& K_{d}=4 \times 10^{-4} \mathrm{~N} \cdot \mathrm{m} \bullet \mathrm{s} / \mathrm{rad} \\
& U_{b a t}=12 \mathrm{v} \\
& R_{a}=4.2 \Omega \\
& K_{m}=0.107 \mathrm{~N} \cdot \mathrm{m} \\
& K_{k}=4.8 \times 10^{-3} \mathrm{~N} \cdot \mathrm{m} \bullet \mathrm{s} / \mathrm{rad}
\end{aligned}
$$

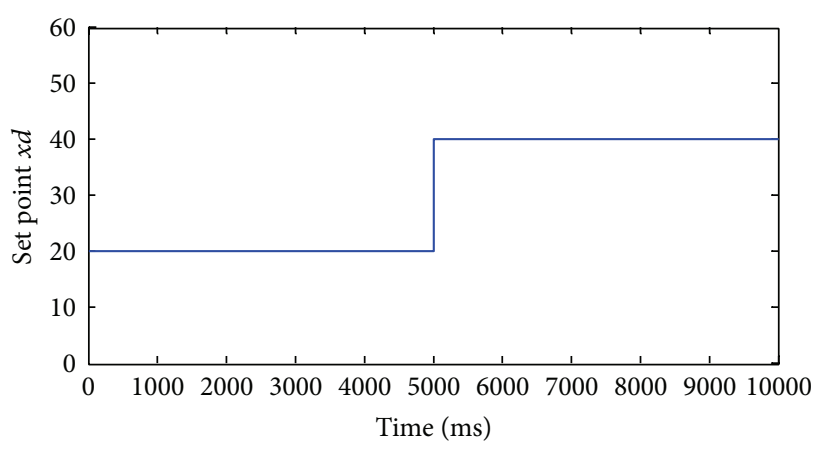

FIGURE 4: Set point of the valve plate angle.

We will analyze the simulation process in detail. During 0-5000 milliseconds, the system is in the steady state and the set point $x_{d}$ is 20 degrees. After 5000 milliseconds, $x_{d}$ is increased from 20 to 40 degrees.

At 5000 milliseconds, $x_{d}$ is increased. In order to increase the actual angle $x_{1}(t)$, the input voltage should be increased. From Figure 9, the duty cycle of the bipolar chopper is increased when time is 5000 milliseconds. The increase of the duty cycle can lead to the increase of the input voltage. When the input voltage is increased, the angle speed $x_{2}(t)$ will be increased, which is shown in Figure 6. When the angle speed $x_{2}(t)$ is increased, the actual angle of the electronic throttle $x_{1}(t)$ will be also increased, which is shown in Figure 5. Therefore, the actual angle $x_{1}(t)$ is regulated to track its set point. When the dynamical regulation process is finished, the angle speed $x_{2}(t)$ is controlled to zero.

During the simulation process, we must give some assumption values for those unknown parameters. So, the values of $\mu_{1}$ and $\mu_{2}$ are known. From Figures 7-8, we also know that the estimation value of the unknown parameters $\widehat{\mu}_{1}$ and $\widehat{\mu}_{2}$ can be adjusted to achieve their actual values $\mu_{1}$ and $\mu_{2}$ by using the adaptive laws shown in (27)-(28).

Therefore, from Figures 4-9, we know that the dynamical process of the simulation result is right for the electronic throttle and the tracking performance is also satisfactory.

\section{Conclusions}

In this paper, the nonlinear dynamical mechanism model and the state-space model of the electronic throttle are presented. 


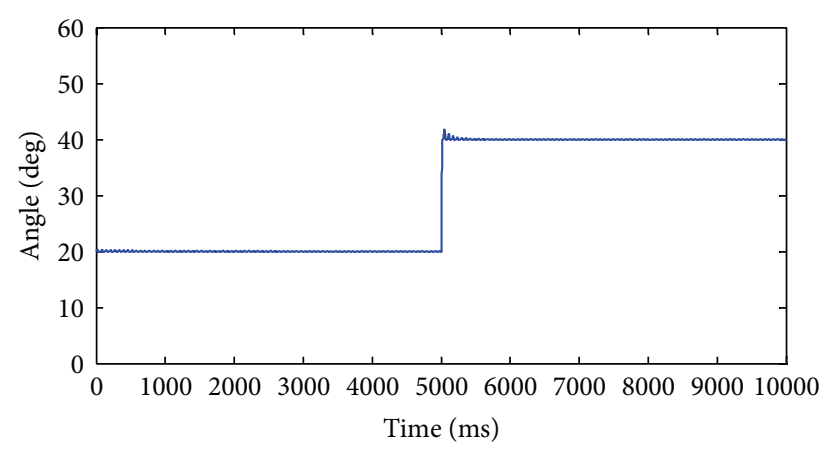

Figure 5: Actual angle of the valve plate.

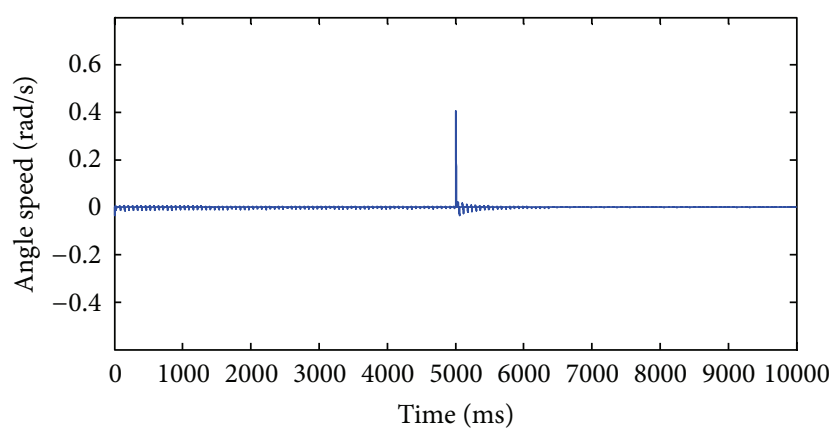

Figure 6: Angle speed of the valve plate.

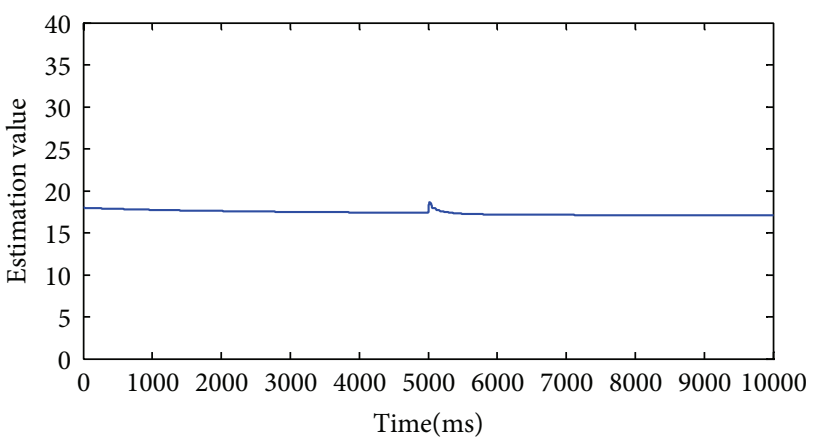

FiguRE 7: Estimation value of the first unknown parameter.

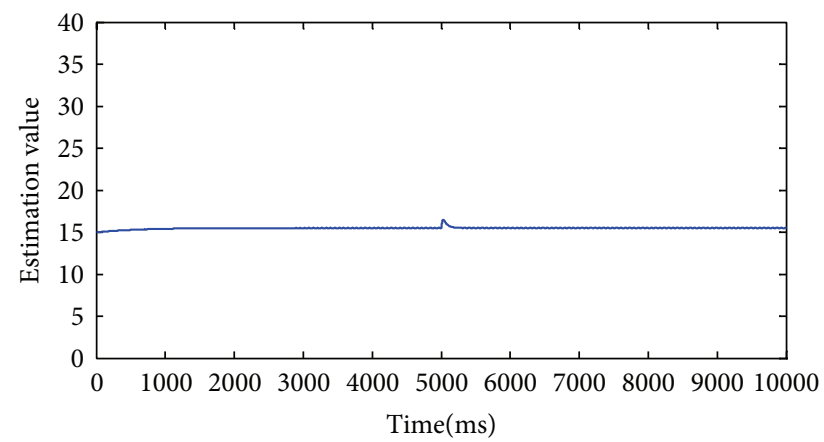

FIGURE 8: Estimation value of the second unknown parameter.

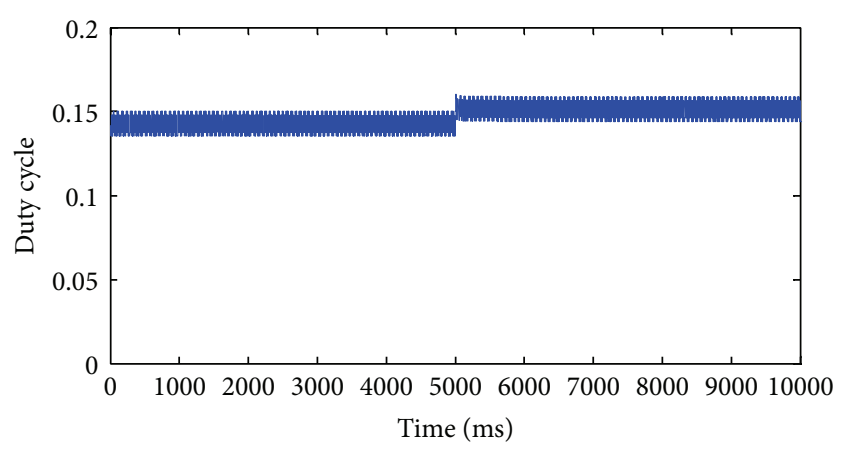

FIGURE 9: Input variable $u(t)$.

According to the dynamical characteristics of the electronic throttle, a new adaptive backstepping sliding-mode controller is developed for the electronic throttle. Parameter adaptive laws are designed to estimate the unknown parameters and sliding-mode control term is applied to compensate the unknown disturbance. Computer simulation results confirm the effectiveness of the proposed control method. The main work mentioned above has certain merit for the modern automobile industry.

\section{Conflict of Interests}

None of the authors of the paper has declared any conflict of interests.

\section{Acknowledgments}

This work was supported by the National Natural Science Foundation of China (no. 61374113) and supported by State Key Laboratory of Synthetical Automation for Process Industries.

\section{References}

[1] P. Stewart and P. J. Fleming, "Drive-by-wire control of automotive driveline oscillations by response surface methodology," IEEE Transactions on Control Systems Technology, vol. 12, no. 5, pp. 737-741, 2004.

[2] C. H. Wang and D. Y. Huang, "A new intelligent fuzzy controller for nonlinear hysteretic electronic throttle in modern intelligent automobiles," IEEE Transaction Industrial Electronics, vol. 60, no. 6, pp. 2332-2345, 2013.

[3] H. K. Khalil, Nonlinear Systems, Prentice Hall, 3rd edition, 2002.

[4] A. Isidori, Nonlinear Control Systems II, Springer, London, UK, 1999.

[5] G. Chen and Z. Han, "Robust right coprime factorization and robust stabilization of nonlinear feedback control systems," IEEE Transactions on Automatic Control, vol. 43, no. 10, pp. 1505-1510, 1998.

[6] N. Bu and M. Deng, "System design for nonlinear plants using operator-based robust right coprime factorization and isomorphism," IEEE Transactions on Automatic Control, vol. 56, no. 4, pp. 952-957, 2011. 
[7] M. Deng, N. Bu, and A. Inoue, "Output tracking of nonlinear feedback systems with perturbation based on robust right coprime factorization," International Journal of Innovative Computing, Information and Control B, vol. 5, no. 10, pp. 33593366, 2009.

[8] X. Yu and O. Kaynak, "Sliding-mode control with soft computing: a survey," IEEE Transactions on Industrial Electronics, vol. 56, no. 9, pp. 3275-3285, 2009.

[9] S. Tong, T. Wang, Y. Li, and B. Chen, "A combined backstepping and stochastic small-gain approach to robust adaptive fuzzy output feedback control," IEEE Transactions on Fuzzy Systems, vol. 21, no. 2, pp. 314-324, 2013.

[10] M. Vašak, M. Baotić, I. Petrović, and N. Perić, "Hybrid theorybased time-optimal control of an electronic throttle," IEEE Transaction Industrial Electronics, vol. 54, no. 3, pp. 1483-1494, 2008.

[11] D. Kim, H. Peng, S. Bai, and J. M. Maguire, "Control of integrated powertrain with electronic throttle and automatic transmission," IEEE Transactions on Control Systems Technology, vol. 15, no. 3, pp. 474-482, 2007.

[12] J. Deur, D. Pavković, N. Perić, M. Jansz, and D. Hrovat, "An electronic throttle control strategy including compensation of friction and limp-home effects," IEEE Transactions on Industry Applications, vol. 40, no. 3, pp. 821-833, 2004.

[13] X. Yuan, Y. Wang, and L. Wu, "SVM-based approximate model control for electronic throttle valve," IEEE Transactions on Vehicular Technology, vol. 57, no. 5, pp. 2747-2756, 2008.

[14] X. Yuan and Y. Wang, "A novel electronic-throttle-valve controller based on approximate model method," IEEE Transactions on Industrial Electronics, vol. 56, no. 3, pp. 883-890, 2009.

[15] T. Aono and T. Kowatari, "Throttle-control algorithm for improving engine response based on air-intake model and throttle-response model," IEEE Transactions on Industrial Electronics, vol. 53, no. 3, pp. 915-921, 2006.

[16] Y. Feng, X. H. Yu, and F. L. Han, "High-order terminal slidingmode observer for parameter estimation of a permanentmagnet synchronous motor," IEEE Transactions on Industrial Electronics, vol. 60, no. 10, pp. 4272-4280, 2013.

[17] C. Rossi, A. Tilli, and A. Tonielli, "Robust control of a throttle body for drive by wire operation of automotive engines," IEEE Transactions on Control Systems Technology, vol. 8, no. 6, pp. 993-1002, 2000.

[18] Y. Pan, Ü. Özguner, and O. H. Dağci, "Variable-structure control of electronic throttle valve," IEEE Transactions on Industrial Electronics, vol. 55, no. 11, pp. 3899-3907, 2008.

[19] A. Beghi, L. Nardo, and M. Stevanato, "Observer-based discretetime sliding mode throttle control for drive-by-wire operation of a racing motorcycle engine," IEEE Transactions on Control Systems Technology, vol. 14, no. 4, pp. 767-775, 2006.

[20] K. Nakano, U. Sawut, K. Higuchi, and Y. Okajima, "Modelling and observer-based sliding-mode control of electronic throttle systems," ECTI Transactions on Electrical Engineering, Electronics, and Communication, vol. 4, no. 1, pp. 22-28, 2006.

[21] M. Barić, I. Petrović, and N. Perić, "Neural network-based sliding mode control of electronic throttle," Engineering Applications of Artificial Intelligence, vol. 18, no. 8, pp. 951-961, 2005.

[22] M. Reichhartinger and M. Horn, "Application of higher order sliding-mode concepts to a throttle actuator for gasoline engines," IEEE Transactions on Industrial Electronics, vol. 56, no. 9, pp. 3322-3329, 2009.
[23] T. Wang, S. Tong, and Y. Li, "Robust adaptive fuzzy control for nonlinear system with dynamic uncertainties based on backstepping," International Journal of Innovative Computing, Information and Control, vol. 5, no. 9, pp. 2675-2688, 2009.

[24] S. Tong, X. He, and H. Zhang, "A combined backstepping and small-gain approach to robust adaptive fuzzy output feedback control," IEEE Transactions on Fuzzy Systems, vol. 17, no. 5, pp. 1059-1069, 2009.

[25] F. Lin, C. Chang, and P. Huang, "FPGA-based adaptive backstepping sliding-mode control for linear induction motor drive," IEEE Transactions on Power Electronics, vol. 22, no. 4, pp. 1222-1231, 2007.

[26] B. Chen and X. Liu, "Fuzzy approximate disturbance decoupling of MIMO nonlinear systems by backstepping and application to chemical processes," IEEE Transactions on Fuzzy Systems, vol. 13, no. 6, pp. 832-847, 2005.

[27] Y. M. Li, S. C. Tong, and T. S. Li, "Adaptive fuzzy output feedback control for a single-link flexible robot manipulator driven DC motor via backstepping," Nonlinear Analysis: Real World Applications, vol. 14, no. 1, pp. 483-494, 2013.

[28] R. Chen, L. Mi, and W. Tan, "Adaptive fuzzy logic based sliding mode control of electronic throttle," Journal of Computational Information Systems, vol. 8, pp. 3253-3260, 2012. 


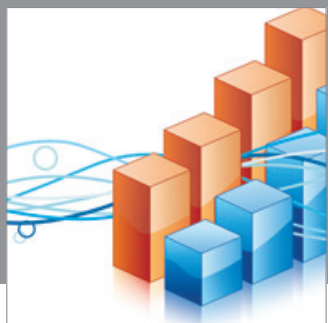

Advances in

Operations Research

mansans

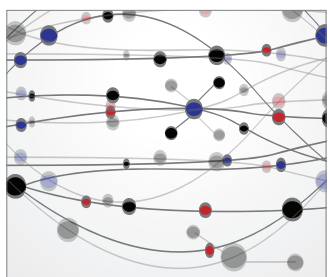

The Scientific World Journal
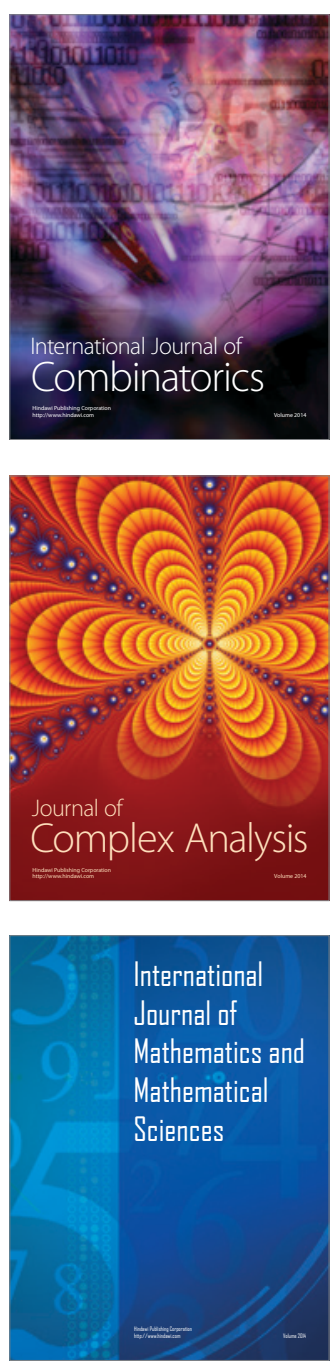
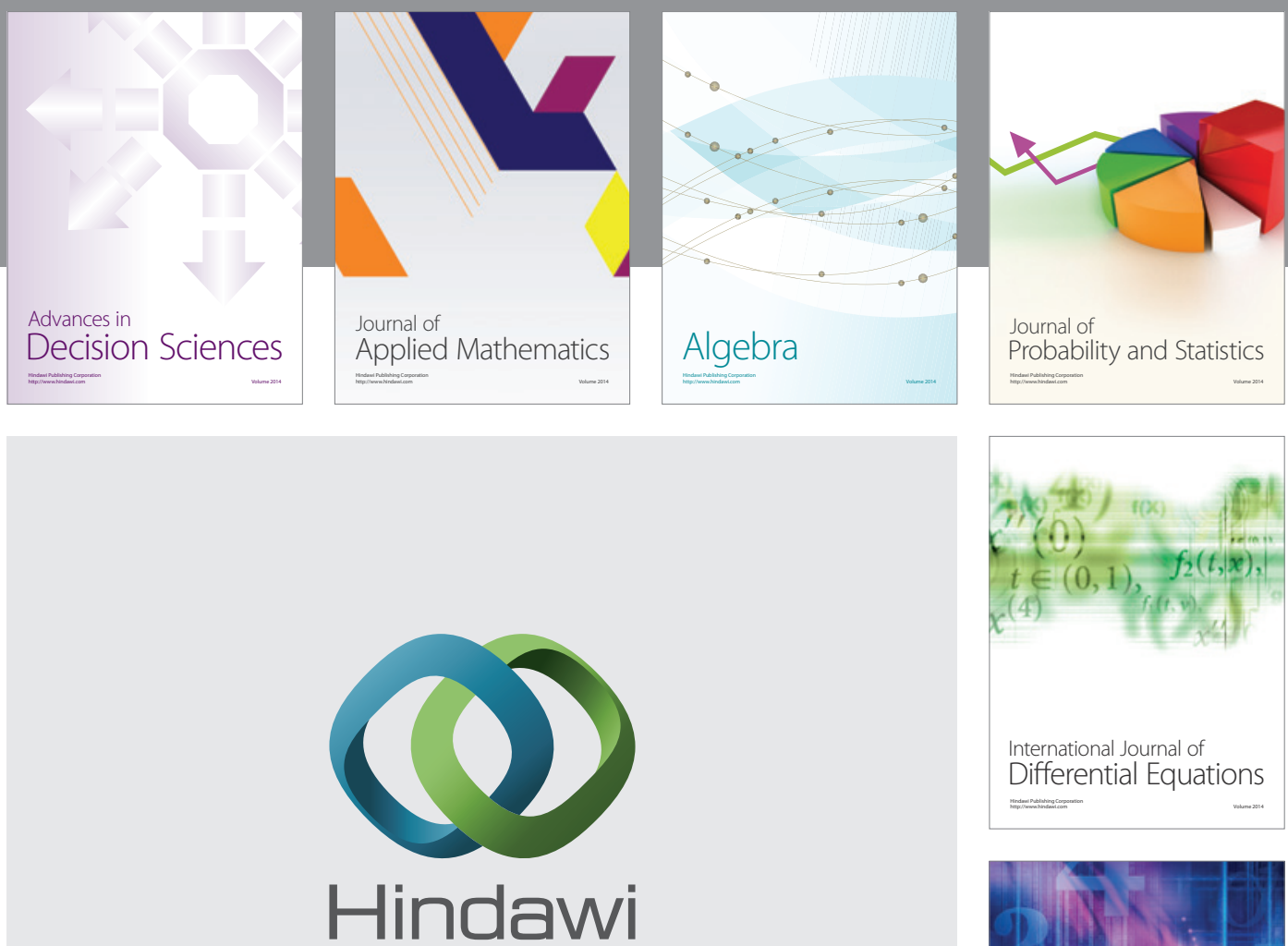

Submit your manuscripts at http://www.hindawi.com
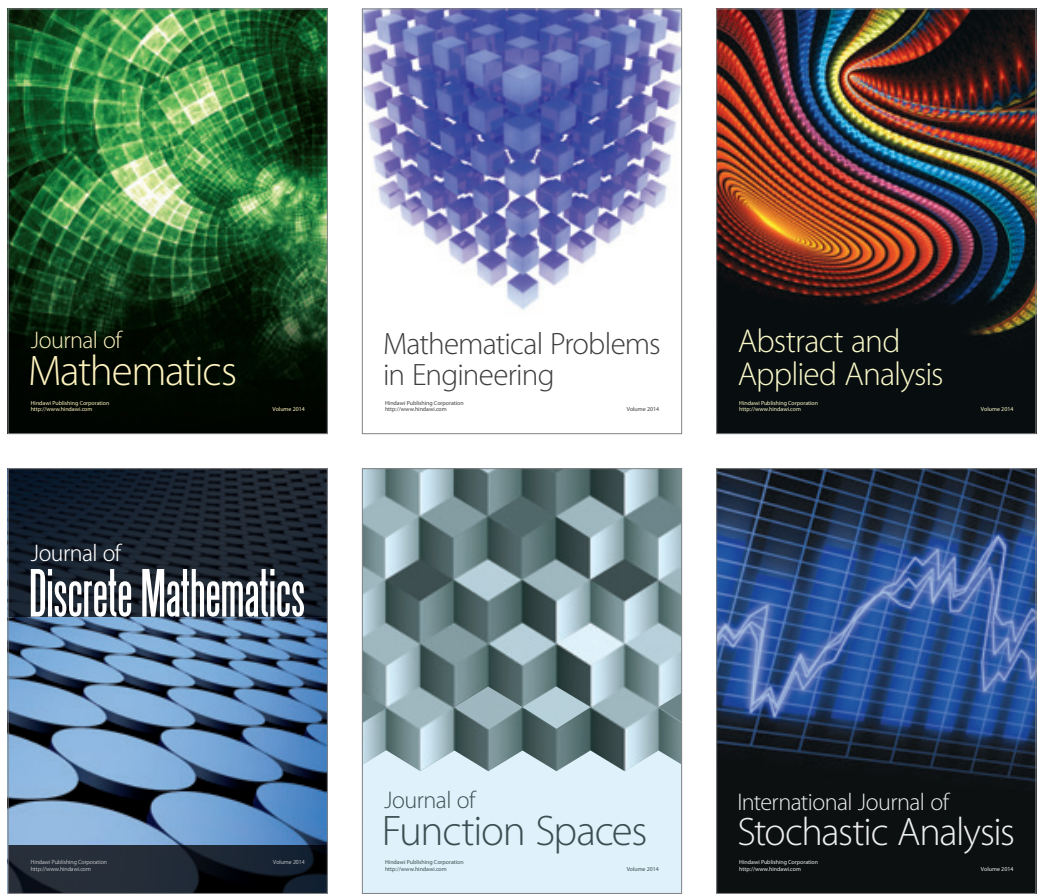

Journal of

Function Spaces

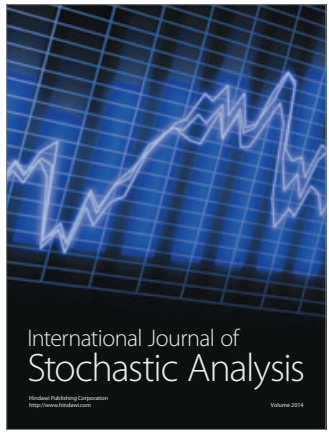

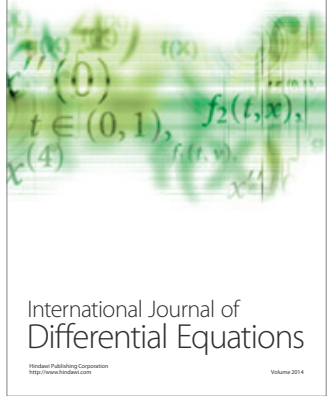
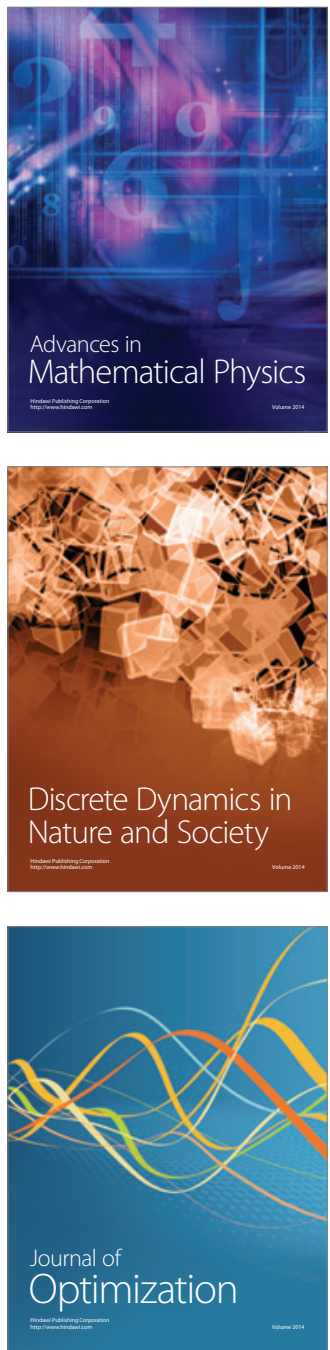